\title{
Kaiso regulates osteoblast differentiation and mineralization via the Itga10/PI3K/AKT signaling pathway
}

\author{
WENWEN TONG ${ }^{*}$, JIA LI $^{*}$, XINZHE FENG ${ }^{*}$, CHEN WANG, YIHONG XU, CHONGRU HE and WEIDONG XU \\ Department of Joint Bone Disease Surgery, Changhai Hospital, \\ Second Military Medical University, Shanghai 200433, P.R. China
}

Received February 19, 2020; Accepted October 30, 2020

DOI: $10.3892 /$ ijmm.2021.4874

\begin{abstract}
Bone homeostasis is maintained by a dynamic balance between bone formation and bone resorption. The cellular activities of osteoblasts and osteoclasts are the primary factors that maintain this dynamic balance. The transcription factor Kaiso has been identified as a regulator of cell proliferation and differentiation in various cells. However, research into its role in bone homeostasis is currently lacking. In the present study, cell and animal experiments were conducted to investigate the role of Kaiso in bone homeostasis. The present study identified that Kaiso was downregulated during osteoblast differentiation in MC3T3-E1 cells. Gain- and loss-of-function studies in MC3T3-E1 cells demonstrated that Kaiso served a critical role in osteoblast differentiation in vitro. The findings were further confirmed in vivo. The results of the sequence analysis indicated that Kaiso influenced osteoblast differentiation and mineralization by regulating the PI3K/AKT signaling pathway. Moreover, integrin subunit $\alpha 10$ (Itga10) was identified as a direct target of Kaiso via chromatin immunoprecipitation and luciferase reporter assays. Collectively, these findings suggested that Kaiso regulated the differentiation of osteoblasts via the Itga10/PI3K/AKT pathway, which represents a therapeutic target for bone formation or bone resorption-related diseases.
\end{abstract}

\section{Introduction}

During the process of bone development and remodeling, there is a dynamic balance between bone formation and bone

Correspondence to: $\mathrm{Dr}$ Chongru $\mathrm{He}$ or $\mathrm{Dr}$ Weidong $\mathrm{Xu}$, Department of Joint Bone Disease Surgery, Changhai Hospital, Second Military Medical University, 168 Changhai Road, Shanghai 200433, P.R. China

E-mail: hechongru@163.com

E-mail: xuweidongjoint@126.com

*Contributed equally

Key words: kaiso, transcription factor, osteoblast differentiation, integrin subunit $\alpha 10$, PI3K/AKT signaling pathway resorption $(1,2)$. When this balance is disrupted, progressive osseous hyperplasia or osteoporosis eventually occur (3). Several diseases are caused by imbalance of bone formation and bone resorption. For instance, excessive bone formation can lead to joint ankylosis in ankylosing spondylitis and osteophyte in osteoarthritis (4,5). Moreover, excessive bone resorption in pathological conditions, such as immobility and corticosteroid overuse, can lead to osteoporosis (6). Axial spondyloarthritis (axSpA) is a chronic inflammatory disease characterized by structural damage which incorporates aspects of bone destruction and new bone formation (4).

The cellular activities of osteoblasts and osteoclasts are the main factors that maintain the dynamic bone balance; osteoblasts are responsible for bone formation, while osteoclasts are responsible for bone resorption $(1,2)$. The cellular activities of osteoblasts are controlled by external signals. For example, bone morphogenetic proteins, a group of molecules belonging to the TGF- $\beta$ superfamily, can induce the differentiation of osteoblasts (6). Another family of growth factors that are implicated in osteoblast differentiation is the Wnt protein family (7). Several transcription factors have also been identified to engage in the process of osteoblast differentiation, including RUNX family transcription factor 2, Osterix and activating transcription factor 4 (8). The transcription factor Kaiso, which is a unique member of the poxviruses and zinc finger (POZ) family, exhibits dual-specificity DNA binding at methylated CpG dinucleotides or at a non-methylated sequence, known as the Kaiso binding site (KBS) $(9,10)$. Kaiso has diverse functions in the regulation of inflammation, cell proliferation and cell cycle (11-15). Several studies have revealed that Kaiso has an effect on Wnt signaling, which is critical in the regulation of bone homeostasis (16-18). However, to the best of our knowledge, no study has investigated the role of Kaiso in osteoblast differentiation and bone formation to date.

In the present study, a HuProt microarray was used to identify autoantibodies in patients with axSpA. Gain- and loss-of-function studies in MC3T3-E1 cells demonstrated that Kaiso served a critical role in osteoblast differentiation in vitro. The findings were further confirmed in vivo. An Illumina HiSeq sequencer was used to analyze the mechanism of Kaiso in regulating osteoblast differentiation and mineralization. Taken together, the present results suggested that Kaiso regulated the differentiation of osteoblasts via the Itga10/PI3K/AKT signaling pathway. 


\section{Materials and methods}

Biologic samples. The study was approved by the Medical Ethics Committee of Changhai Hospital (approval no. CHEC2017-163) and written informed consent was obtained from all patients (age, 28.57 \pm 3.95 years; 5 men; 2 women). Serum samples used for the protein chip were obtained in patients (seven patients with axSpA vs. seven healthy controls) recruited from the outpatient clinic of Changhai Hospital between 2018.01 and 2019.01. The HuProt microarrays used in this study were provided by CDI Laboratories, Inc. The array contained duplicate spots of 20,000 individually purified human proteins with an N-terminal glutathione S-transferase tag.

Bone marrow mesenchymal stem cells were extracted from six patients with femoral neck fractures who underwent surgical treatment recruited from the Changhai Hospital between 2018.01 and 2019.01 .

Cell culture. The MC3T3-E1 (mouse pre-osteoblast) cell line was obtained from The Cell Bank of Type Culture Collection of Chinese Academy of Science and grown in $\alpha$-minimal essential medium (Gibco; Thermo Fisher Scientific, Inc.) supplemented with 10\% FBS (Gibco; Thermo Fisher Scientific, Inc.), $100 \mathrm{U} / \mathrm{ml}$ penicillin and $0.1 \mathrm{mg} / \mathrm{ml}$ streptomycin (Gibco; Thermo Fisher Scientific, Inc.). To induce mineralization, MC3T3-E1 cells were treated for different time points $(1,3$, $5,7,14$ and 21 days) with osteogenic medium (OM), which was composed of complete medium supplemented with $50 \mu \mathrm{g} / \mathrm{ml}$ ascorbic acid (Sigma-Aldrich; Merck KGaA), $10 \mathrm{mM} \beta$-glycerophosphate (Sigma-Aldrich; Merck KGaA) and $50 \mathrm{ng} / \mathrm{ml} \mathrm{BMP2}$ (Novus Biologicals, LLC) in a humidified incubator at $37^{\circ} \mathrm{C}$ under $5 \% \mathrm{CO}_{2}$. The medium was changed every 3 days. To validate the role of the PI3K/AKT signaling pathway in osteogenic differentiation, $15 \mathrm{mM}$ PI3K inhibitor (LY294002; Selleck Chemicals) was added to the $\mathrm{OM}\left(4^{\circ} \mathrm{C}\right.$ for 3 days) and the medium changed every 3 days.

Preparation and transfection of lentivirus. For gene overexpression, a lentivirus expression vector was constructed by inserting full-length Kaiso cDNA into the pLenti-EF1aEGFP-F2A-Puro-CMV-MCS vector (Obio Technology Co., Ltd.), to generate LV-Kaiso. Empty vector plasmid was used as control. For gene silencing, Kaiso-targeting short hairpin RNA (shRNA) or Itga10-targeting shRNA were inserted into the PLKD-CMV-EGFP-Puro-U6 vector (Obio Technology Co., Ltd.). The sequences of the shRNAs are summarized in Table SI (supplied by Obio Technology Co., Ltd.). The reconstructed vectors were then transfected into 293T cells, and the lentiviral particles were collected at $48 \mathrm{~h}$ post-transfection. The construction and production of the lentivirus were performed by Obio Technology Co., Ltd. MC3T3-E1 cells were infected with viruses (at a multiplicity of infection of 50) in the presence of $5 \mu \mathrm{g} / \mathrm{ml}$ polybrene $\left(37^{\circ} \mathrm{C}\right.$ for $24 \mathrm{~h}$ ), and replaced it with fresh medium after $24 \mathrm{~h}$. After $72 \mathrm{~h}$ of infection, the fluorescence expression was observed by fluorescence microscope at a magnification of $x 100$. Then the cells were selected using puromycin $(2 \mu \mathrm{g} / \mathrm{ml})$ to establish stable cell lines. Small interfering (si)RNAs were transfected using Lipofectamine ${ }^{\circledast}$ RNAiMAX (Invitrogen; Thermo Fisher Scientific, Inc.). The sequences of the siRNAs are summarized in Table SI.
RNA extraction and reverse transcription-quantitative PCR $(R T-q P C R)$. Total RNA was extracted using TRIzol ${ }^{\circledR}$ reagent (Invitrogen; Thermo Fisher Scientific, Inc.), and RT $\left(37^{\circ} \mathrm{C}\right.$ for $15 \mathrm{~min}$ and $85^{\circ} \mathrm{C}$ for $5 \mathrm{sec}$, and cooled to $4^{\circ} \mathrm{C}$ ) was performed with a PrimeScript RT Reagent kit (Takara Bio, Inc.) according to the manufacturer's protocols. RT-qPCR was performed $\left(95^{\circ} \mathrm{C}\right.$ pre-incubation for $5 \mathrm{~min}$, followed by 40 cycles at $95^{\circ} \mathrm{C}$ for $20 \mathrm{sec}, 60^{\circ} \mathrm{C}$ for $15 \mathrm{sec}$ and $72^{\circ} \mathrm{C}$ for $20 \mathrm{sec}$, and cooled to $4^{\circ} \mathrm{C}$ ) on an ABI Prism 7500 Sequence Detection system (Applied Biosystems; Thermo Fisher Scientific, Inc.) using SYBR Premix Ex Taq ${ }^{\mathrm{TM}}$ (Takara Bio, Inc.). The relative expression of each target gene was quantified by calculating the quantification cycle value, and was normalized to the levels of $\beta$-actin. The relative expression levels were calculated based on the $2^{-\Delta \Delta C q}$ method (19). Each sample was analyzed in triplicate. The primer sequences are listed in Table SII.

Western blot analysis. Cells were lysed with RIPA buffer supplemented with protease inhibitors (Thermo Fisher Scientific, Inc.). The Pierce ${ }^{\mathrm{TM}}$ Rapid Gold BCA Protein Assay kit (Thermo Fisher Scientific, Inc.) was used for protein determination. Equal quantities of proteins $(20 \mu \mathrm{g})$ were separated by 6 or 10\% SDS-PAGE and transferred to PVDF membranes (EMD Millipore). After pre-incubation in 5\% non-fat milk for $1 \mathrm{~h}$, the membranes were incubated with primary antibodies for $12-16 \mathrm{~h}$ at $4^{\circ} \mathrm{C}$. The membranes were then incubated with appropriate horseradish peroxidase-conjugated secondary antibodies (cat. no. 7074; 1:3,000; Cell Signaling Technology, Inc.) for $1 \mathrm{~h}$ at room temperature. The immunoreactive protein bands were visualized using an enhanced chemiluminescence detection system (Cytiva). The primary antibodies used in this study were as follows: Anti-Kaiso (cat. no. 12723; 1:1,000; Abcam), anti-Itga10 (cat. no. PA5-67829; 1:1,000; Merck KGaA), anti-p85 (cat. no. 4292; 1:1,000; Cell Signaling Technology, Inc.), anti-AKT (cat. no. 9272; 1:1,000; Cell Signaling Technology, Inc.), anti-phosphorylated (p)-AKT (cat. no. 9271; 1:1,000; Cell Signaling Technology, Inc.) and anti- $\beta$-tubulin (cat. no. 2146; 1:1,000; Cell Signaling Technology, Inc.). The relative semi-quantification of western blotting was performed using ImageJ software (1.52a; National Institutes of Health).

Alkaline phosphatase (ALP) activity assay. After treatment with OM for 1, 3, 5 and 7 days, MC3T3-E1 cells were rinsed with PBS, solubilized in lysis buffer $(50 \mathrm{mM}$ Tris, $100 \mathrm{mM}$ glycine) for $5 \mathrm{~min}$ and centrifuged $(2,000 \mathrm{x} \mathrm{g}$; room temperature; $5 \mathrm{~min}$ ) to collect the supernatant. The ALP activity in the supernatant was then determined using ALP Assay kits (Nanjing Jiancheng Bioengineering Institute) according to the manufacturer's instructions. The ALP activity was normalized to total the protein concentration, which was determined using BCA Protein Assay kits (Thermo Fisher Scientific, Inc.).

ALP staining and Alizarin Red S staining. MC3T3-E1 cells were cultured in 24 -well plates at a density of $3 \times 10^{4}$ cells per well. After treatment with $\mathrm{OM}\left(37^{\circ} \mathrm{C}\right)$ for a different numbers of days (1, 3, 5, 7, 14 and 21 days), cells were washed with PBS, fixed with $4 \%$ formaldehyde for $30 \mathrm{~min}$ (room temperature) and rinsed with double-distilled $\mathrm{H}_{2} \mathrm{O}$. For ALP staining, ALP Assay kits (Beyotime Institute of Biotechnology) were 
used according to the manufacturer's protocol. For detection of mineral deposition, the fixed cells were stained with $1 \%$ Alizarin Red S solution (Sigma-Aldrich; Merck KGaA) at room temperature for $10 \mathrm{~min}$. All the stained cells were rinsed three times with double-distilled $\mathrm{H}_{2} \mathrm{O}$ and then imaged using a camera.

Sequencing analysis of $m R N A$. Total RNA was extracted from stable cell lines transfected with LV-ctr, LV-Kaiso, Sh-ctr and Sh-Kaiso-1 using TRIzol reagent. The quantity and purity of total RNA were monitored using a NanoDrop 2000 spectrophotometer (NanoDrop Technologies; Thermo Fisher Scientific, Inc.) and an Agilent 2200 Bioanalyzer (Agilent Technologies, Inc.). Library construction and sequencing on the Illumina HiSeq 2000 platform (Illumina, Inc.) were performed by Guangzhou RiboBio Co., Ltd.

Gene Ontology (GO) (http://geneontology.org/) and Kyoto Encyclopedia of Genes and Genomes (KEGG) pathway analyses (https://www.genome.jp/kegg/) were performed to determine the roles of the differentially expressed genes in biological pathways. Protein-protein interaction (PPI) network analysis (https://string-db.org/) was performed to identify the key genes in the pathways. $\mathrm{P}<0.05$ was considered to indicate statistically significant enrichment.

Chromatin immunoprecipitation (ChIP) assay. JASPAR (http://jaspar.genereg.net/) and MatInspector (https://www. genomatix.de/online_help/help_matinspector/matinspector_ help.html) were used to predict the presence of Kaiso binding site in Itga10 promoter. ChIP assays were performed using an EZ ChIP kit (cat. no. 17-10086; EMD Millipore) according to the manufacturer's protocol. In brief, cells were fixed with $1 \%$ formaldehyde for $10 \mathrm{~min}\left(37^{\circ} \mathrm{C}\right)$ to crosslink chromatin complexes before the addition of $125 \mathrm{mM}$ glycine to stop the reaction. After washing with PBS, the samples were then sonicated at $4^{\circ} \mathrm{C}$ to obtain DNA fragments (400-1,000 bp). The sonicator Bioruptor was used at mid-range and the cell samples were sonicated for $10 \mathrm{~min}$, 'ON' for $30 \mathrm{sec}$, 'OFF' for $30 \mathrm{sec}$ and centrifuged at $4^{\circ} \mathrm{C}$ for $10 \mathrm{~min}$ at $2,000 \mathrm{x}$ g to remove insoluble materials. Immunoprecipitation was performed $\left(4^{\circ} \mathrm{C}\right.$ for $24 \mathrm{~h}$ ) using a Kaiso-specific antibody (cat. no. 12723; 1:1,000; Abcam) or an IgG control antibody. Protein A/G beads (Thermo Fisher Scientific, Inc.) were used to pull down the immune complexes. The protein-DNA complexes were eluted with buffer containing $1 \%$ SDS and $0.1 \mathrm{M} \mathrm{NaHCO}_{3}$, and crosslinks were reversed at $65^{\circ} \mathrm{C}$ overnight. DNA was purified and used for PCR. The following Itga10 primers were used for ChIP assay: Forward, 5'-CCGAATGGAAGATGAGAG ACA-3' and reverse, 5'-TCGGAGGTTAATACCGATGC-3'.

Luciferase reporter assay. To generate the Itga10 luciferase reporter plasmids, a 1,051-bp DNA fragment upstream of the Itga10 transcription start site was amplified via PCR from genomic human DNA and cloned into the pGL4.10-Basic vector (Promega Corporation). The predicted Kaiso binding sites (5'-AATCCTGCTAC-3') and the corresponding mutated Kaiso binding sites (5'-TGGCTCGTGT-3') were also subcloned into the vector using standard cloning strategies (20). For luciferase assays, MC3T3-E1 cells were cultured to 70-80\% confluence in 24-well plates before co-transfection with the luciferase reporter vector $(0.1 \mu \mathrm{g})$ and Kaiso overexpression vectors or control $(0.1 \mu \mathrm{g})$ using Lipofectamine ${ }^{\circledR} 2000$ (Invitrogen; Thermo Fisher Scientific, Inc.) according to the manufacturer's instructions $\left(37^{\circ} \mathrm{C}\right.$ for $\left.48 \mathrm{~h}\right)$. At $48 \mathrm{~h}$ after transfection, the luciferase activity was measured using the Dual Luciferase Reporter Assay system (Promega Corporation), and each experiment was repeated in triplicate. The transfection efficiency was normalized by determining the activity of Renilla luciferase.

Osteogenesis assay in vivo. NOD/SCID mice (male; age, 8 weeks; $18-22 \mathrm{~g} ; \mathrm{n}=21$ ) purchased from SLRC Laboratory. Mice were bred under specific pathogen-free conditions at a constant temperature $\left(23 \pm 1^{\circ} \mathrm{C}\right)$ with $55 \pm 15 \%$ humidity and a 12-h light-dark cycle (09:00-21:00). All the mice had free access to food and water. For the in vivo osteogenic ectopic assay, a total of $2 \times 10^{6} \mathrm{MC} 3 \mathrm{~T} 3$-E1 were seeded on a $\beta$-tricalcium phosphate $(\beta$-TCP) scaffold (Shanghai Bio-lu Biomaterials Co., Ltd.). The implants loaded with cells were implanted into an intramuscular pocket of the right femur of mice (21). The mice were anesthetized with an injection of $1 \%$ pentobarbital sodium $(70 \mathrm{mg} / \mathrm{kg})$ into the abdominal cavity. The mice were randomly divided into seven groups containing three mice per group: i) $\beta$-TCP (vehicle); ii) $\beta$-TCP loading LV-ctr MC3T3-E1 cells (LV-ctr); iii) $\beta$-TCP loading LV-Kaiso MC3T3-E1 cells (LV-Kaiso); iv) $\beta$-TCP loading Sh-ctr MC3T3-E1 cells (Sh-ctr); v) $\beta$-TCP loading Sh-Kaiso MC3T3-E1 cells (Sh-Kaiso); vi) $\beta$-TCP loading Sh-ctr MC3T3-E1 cells (Sh-ctr); and vii) $\beta$-TCP loading Sh-Itga10 MC3T3-E1 cells (Sh-Itga10). At 8 weeks post-implantation, the mice were sacrificed via carbon dioxide asphyxia. Briefly, the $\mathrm{CO}_{2}$ flow rate was started with $8 \mathrm{l} / \mathrm{min}$ in a 30 -lchamber. The implants were harvested and fixed in $4 \%$ paraformaldehyde (room temperature for $24 \mathrm{~h}$ ) for $\mu$-computed tomography $(\mu \mathrm{CT})$ analysis. The bone mineral density (BMD) of implants was then generated by skyscan 1176 (22). The implants were decalcified in 10\% EDTA and embedded in paraffin. Then $5-\mu \mathrm{m}$ sections were stained with hematoxylin and eosin (cat. no. C0105M; Beyotime Institute of Biotechnology) for $10 \mathrm{~min}$ at room temperature, and washed with distilled water for $10 \mathrm{~min}$. Sections were observed and imaged using a light microscope (E200; Nikon Corporation) at a magnification of x200. All animal studies were approved by the Animal Ethics Committee of Changhai Hospital.

Statistical analysis. SPSS software (version 20; IBM Corp.) was used for statistical analysis. Differences between two groups were observed using unpaired Student's t-tests, and differences in the results derived from experiments with $>2$ groups were analyzed using one-way ANOVA followed by Bonferroni post-hoc test. $\mathrm{P}<0.05$ was considered to indicate a statistically significant difference. The experiment was repeated three times independently.

\section{Results}

Autoantibodies in axSpA. The present study used the HuProt microarray to identify autoantibodies in patients with axSpA and healthy controls. The results demonstrated that seven candidate autoantibodies [including microtubule associated protein 9, BAF chromatin remodelling complex subunit 
BCL7A, argonaute RISC component 1, immunoglobulin heavy constant $\gamma 1$ (G1m marker), myosin light chain kinase, SGT1 homolog, MIS12 kinetochore complex assembly cochaperone andKaiso (also known as zinc finger and BTB domain containing 33)] were present specifically in serum from the patients with axSpA but not from that of the healthy controls (Fig. S1).

Kaiso regulates osteoblast differentiation in MC3T3-E1 cells. The expression of Kaiso was found to be downregulated during osteoblast differentiation in MC3T3-E1 cells (Fig. 1A). To investigate the role of Kaiso in the regulation of osteoblast differentiation, MC3T3-E1 cells were infected with a lentivirus expressing Kaiso or control virus to produce a stable overexpressing cell line (LV-Kaiso) or control cell line (LV-ctr). Stable overexpression of Kaiso was confirmed via western blot analyses and qPCR (Fig. 1B and C). To examine the osteoblast differentiation ability, the stable cell lines were cultured for the indicated periods in OM, and mRNA expression of the osteogenic marker genes, including ALP, osteocalcin (OCN) and bone sialoprotein (BSP), were examined. The mRNA expression levels of ALP, OCN and BSP were significantly decreased in the LV-Kaiso group compared with those in LV-ctr group after treatment with OM for 7 days (Fig. 1D). Moreover, ALP activity assays and ALP staining demonstrated that Kaiso overexpression suppressed ALP activity in MC3T3-E1 cells after induction by OM for different periods (Fig. 1E and F). The effects of Kaiso on late osteoblast differentiation were examined using Alizarin Red S staining to assess mineral bone nodule formation. The Alizarin Red staining results further indicated that Kaiso overexpression notably suppressed the osteogenic differentiation of MC3T3-E1 cells (Fig. 1G).

To further clarify the role of Kaiso in osteoblast differentiation, Kaiso knockdown was conducted in MC3T3-E1 cells using shRNA (Sh-Kaiso-1 andSh-Kaiso-2). Control cells were also prepared with Sh-ctr (Fig. 1H and I). By contrast to those in Sh-ctr cells, the mRNA expression levels of ALP, OCN and BSP were increased in Sh-Kaiso MC3T3-E1 cells (Fig. 1J). As Sh-Kaiso-1 was more active, this transfectant was used for further experiments. ALP activity assays, ALP staining and Alizarin Red S staining at different timepoints demonstrated stronger osteogenic differentiation of Sh-Kaiso MC3T3-E1 cells compared with that of Sh-ctr MC3T3-E1 cells (Fig. 1K-M). Similar results were observed in primary human bone marrow-derived mesenchymal stem cells (Fig. S2). The expression of Kaiso was also found to be downregulated during osteoblast differentiation in primary human bone marrow-derived mesenchymal stem cells (BMSCs). The mRNA expression levels of ALP, OCN and BSP were increased after knockdown of Kaiso in BMSCs (Fig. S2). These results suggested that Kaiso negatively regulated osteoblast differentiation.

Kaiso regulates osteoblast differentiation by modulating the PI3K/AKT signaling pathway in MC3T3-E1 cells. To gain insights into the molecular mechanisms via which Kaiso regulates osteoblast differentiation, the Illumina HiSeq sequencer was used to analyze changes in mRNA expression in Kaiso-overexpressing or Kaiso-knocked down stable cell lines. GO analysis of biological processes demonstrated that the downregulated genes in LV-Kaiso cells and the upregulated genes in Sh-Kaiso cells were significantly enriched in 'osteoblast differentiation' (Fig. 2A and C). KEGG pathway analysis of the differentially expressed genes identified that the 'PI3K/AKT pathway' was inhibited in LV-Kaiso MC3T3-E1 cells and enhanced in Sh-Kaiso MC3T3-E1 cells (Fig. 2B and D). PPI network analysis of the differentially expressed genes enriched in the PI3K/AKT pathway indicated that Itga10, colony stimulating factor 1 receptor (Csf1r) andfibroblast growth factor receptor 3 (Fgfr3), with higher degrees of connectivity than other genes, were network-centric proteins (Fig. 2E).

To confirm the ability of Kaiso in regulating the PI3K/AKT pathway, the expression levels of p85 and p-AKT were determined via western blot analysis. The results demonstrated that the expression levels of p85 and p-AKT were decreased in LV-Kaiso MC3T3-E1 cells and increased in Sh-Kaiso MC3T3-E1 cells (Fig. 3A). To further evaluate the involvement of the PI3K/AKT signaling pathway in the enhanced osteoblast differentiation observed in Sh-Kaiso MC3T3-E1 cells, the PI3K-specific inhibitor LY294002 was added into the cell culture, and its effect on osteoblast differentiation was examined. LY294002 markedly blocked the enhancement of p-AKT and p85 protein expression levels observed in Sh-Kaiso MC3T3-E1 cells (Fig. 3A). The intensity of p85 and p-AKT was determined via densitometry using Image J software and normalized to the loading control $\beta$-tubulin (Fig. S3). LY294002 also had significant effects on osteoblast differentiation, as demonstrated by the decreased mRNA expression levels of ALP, OCN and BSP (Sh-ctr vs. Sh-ctr+LY; Sh-Kaiso-1vs. Sh-Kaiso-1+LY;Sh-ctr+LY vs. Sh-Kaiso-1+LY) (Fig. 3B-D). The results of ALP activity assays, ALP staining and Alizarin Red $S$ staining further demonstrated that LY294002 inhibited the increased ALP activity and mineral bone nodule formation in Sh-Kaiso MC3T3-E1cells (Sh-ctr vs. Sh-ctr+LY; Sh-Kaiso-1 vs. Sh-Kaiso-1+LY; Sh-ctr+LY vs. Sh-Kaiso-1+LY) (Fig. 3E and F). Thus, these findings indicated that Kaiso regulated the osteoblast differentiation of MC3T3-E1 cells via a mechanism involving the PI3K/AKT pathway.

Kaiso regulates the PI3K/AKT pathway via Itgal0. To further investigate the mechanism via which Kaiso regulates the PI3K/AKT pathway, the present study focused on Itga10, Csf1r and Fgfr3, which demonstrated a high degree of connectivity in the PPI network analysis. The results of the western blot analysis indicated that Itga10 was downregulated in LV-Kaiso MC3T3-E1 cells and upregulated in Sh-Kaiso MC3T3-E1 cells (Fig. 4A and B). The existence of the KBS (5'-TCCTGCNA-3') in the promoter region of Itga10 (5'-AATCCTGCTAC-3') was predicted using JASPAR and MatInspector databases. For further verification, ChIP assays were performed on LV-Kaiso and control LV-ctr cell lines using an anti-Kaiso monoclonal antibody. qPCR of the ChIP DNA products using an Itga10 promoter primer set demonstrated the binding of Kaiso to the Itga10 promoter (Fig. 4C). To further assess these findings, the promoter activity of wild-type (WT) and KBS-mutated (MUT) Itga10 was detected in MC3T3-E1 cells. It was identified that Kaiso overexpression significantly inhibited the promotor luciferase reporter activity of Itga10. Moreover, the MUT of 

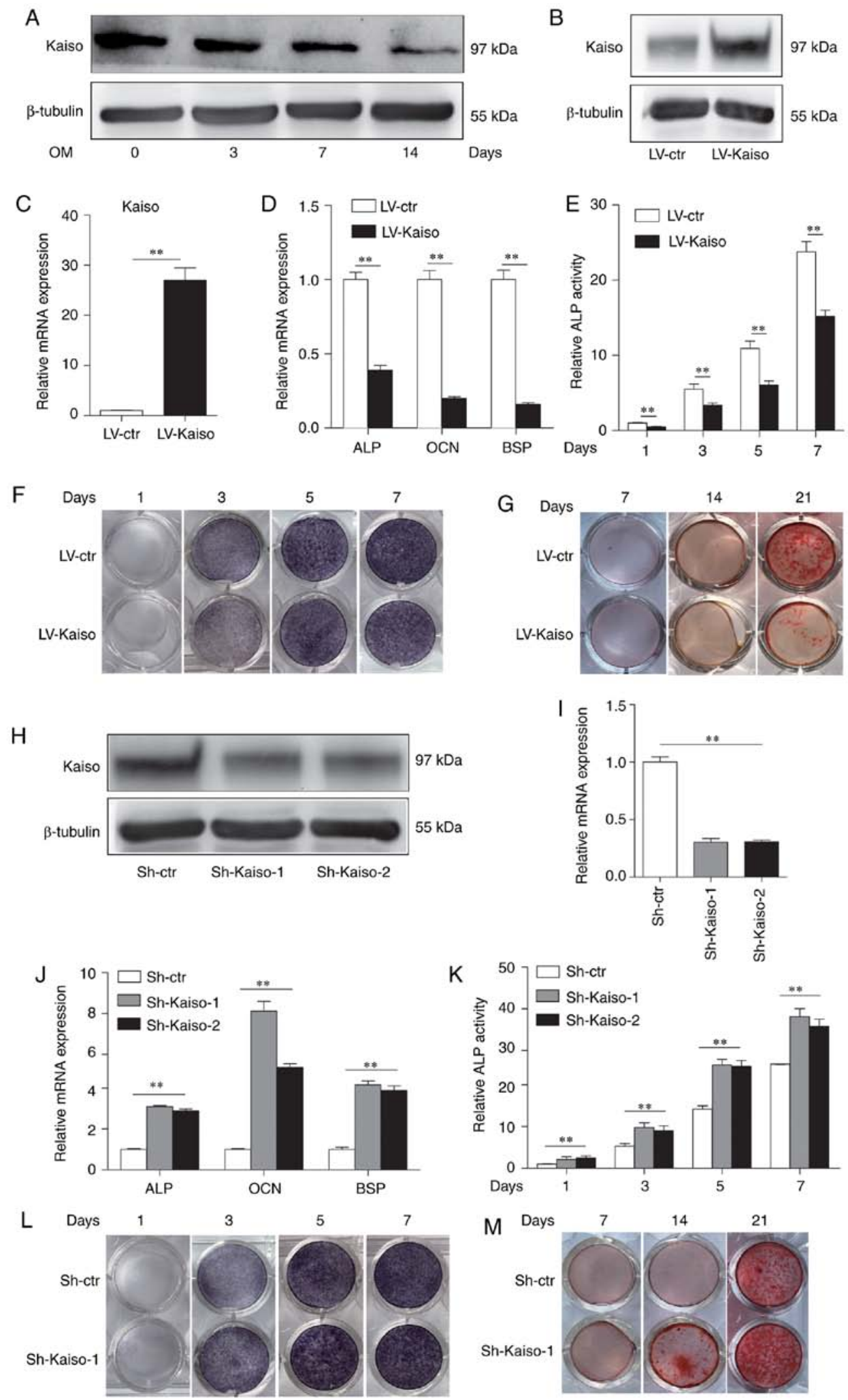

Figure 1. Overexpression or knocking down of Kaiso can inhibitor promote osteoblast differentiation in MC3T3-E1 cells. (A) Kaiso expression during osteogenic induction was determined via western blot analysis. (B) Western blot analysis and (C) RT-qPCR of Kaiso expression in LV-Kaiso and LV-ctr infected MC3T3-E1 cells. (D) RT-qPCR analysis of ALP, OCN and BSP expression levels was performed in LV-Kaiso and LV-Ctr infected MC3T3-E1 cells after 7 days of culture with OM. (E) ALP activity was measured on days 1,3,5 and 7 after culture with OM. The results are presented as the value relative to that at day 1 . (F) ALP staining was performed on days 1,3,5 and 7 in the presence of OM. (G) Mineralized nodule formation was detected with Alizarin Red S staining on days 7, 14 and 21. (H) Western blot analysis and (I) RT-qPCR analysis of Kaiso expression in Sh-Kaiso-1, Sh-Kaiso-2 and Sh-ctr transfected MC3T3-E1 cells. (J) RT-qPCR analysis of ALP, OCN and BSP expression levels was performed in Sh-ctr and Sh-Kaiso transfected MC3T3-E1 cells after 7 days of culture with OM. (K) ALP activity. (L) ALP staining. (M) Mineralized nodule formation. All the data were analyzed in triplicate. Data are presented as the mean $\pm \mathrm{SD}$. ${ }^{* *} \mathrm{P}<0.01$. $\mathrm{P}$-values of C-E were based on Student's t-test and P-values of I-K were analyzed using one-way ANOVA. RT-qPCR, reverse transcription-quantitative PCR; OM, osteogenic medium; ALP, alkaline phosphatase; OCN, osteocalcin; BSP, bone sialoprotein; LV, lentivirus; shRNA, short hairpin RNA; Ctr, control. 
A
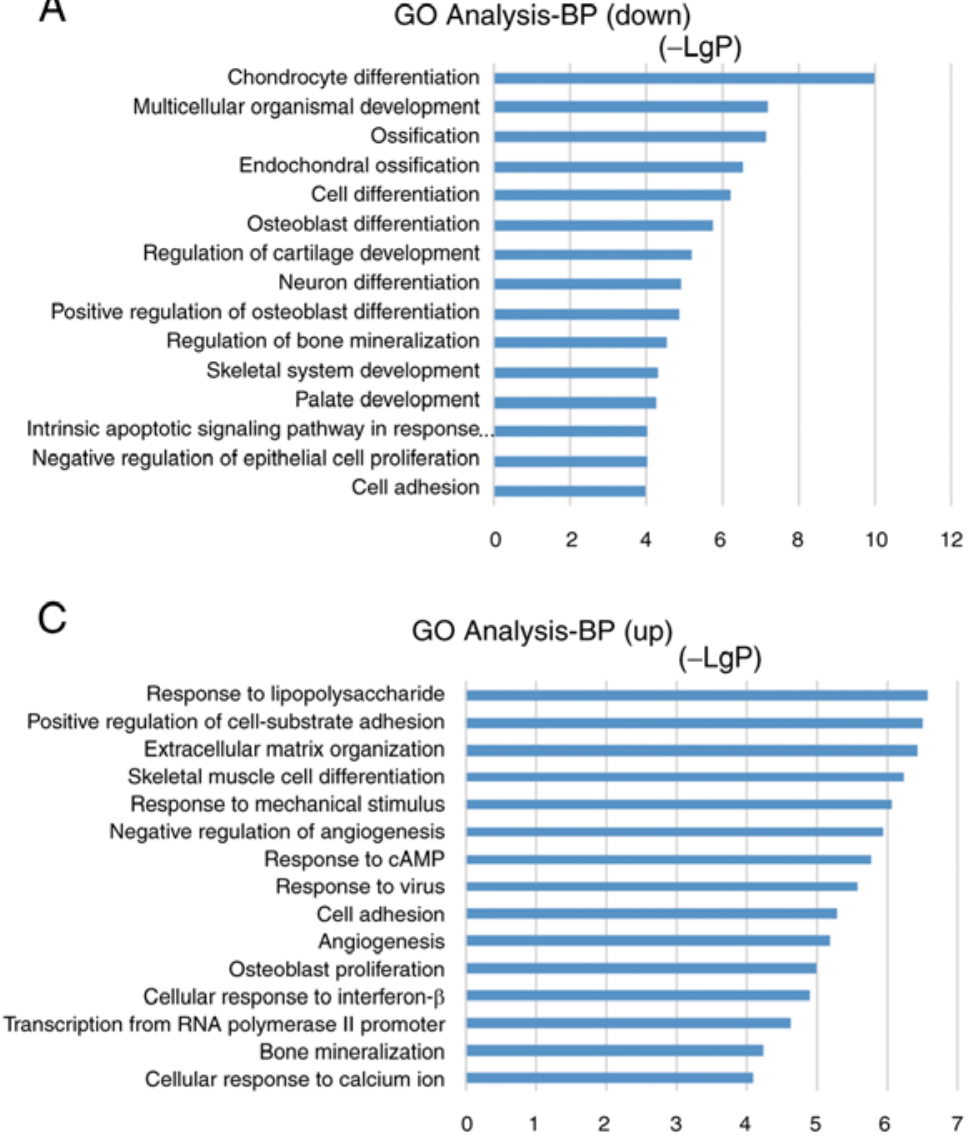

E

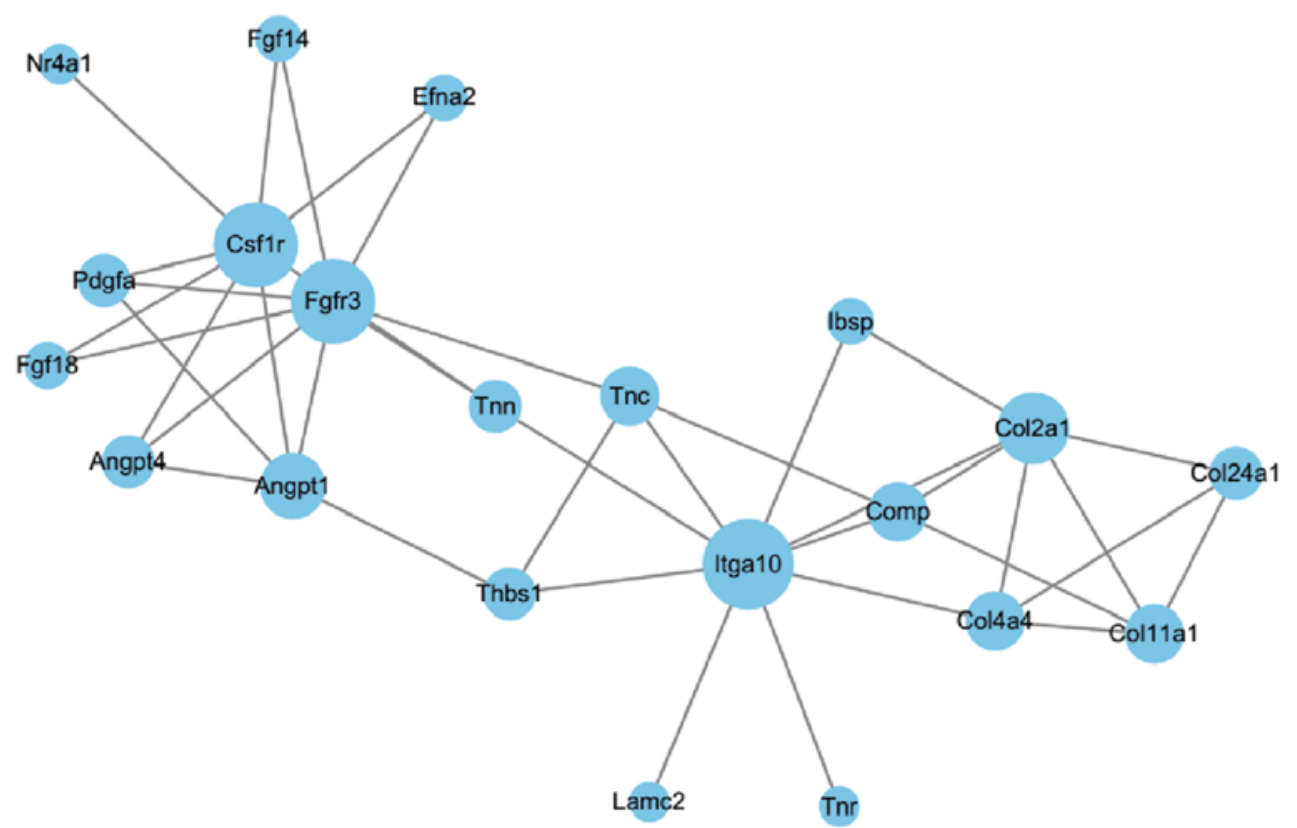

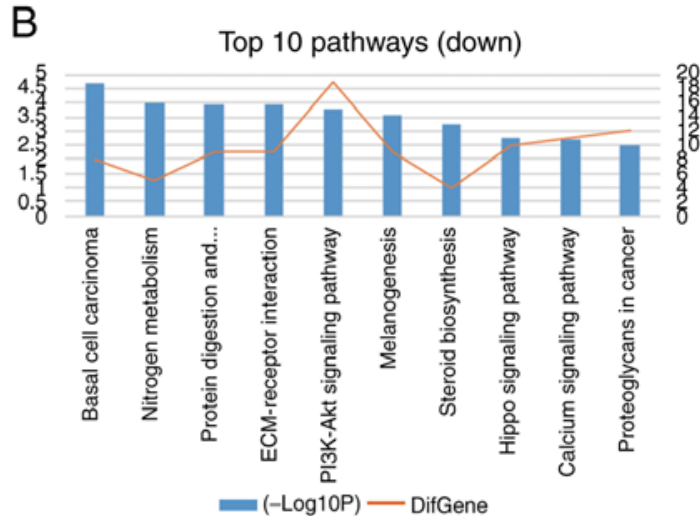

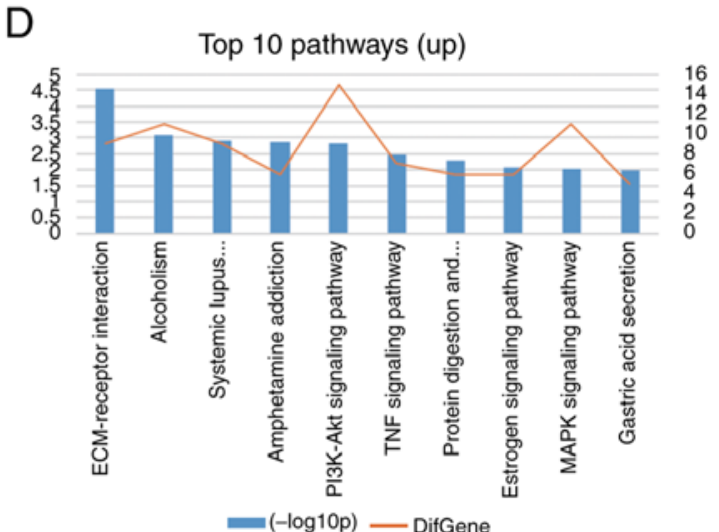

Figure 2. mRNA sequence analysis in LV-Kaiso and Sh-Kaiso infected MC3T3-E1 cells. (A) GO analysis of BP in downregulated genes in LV-Kaiso MC3T3-E1 cells vs. LV-ctr MC3T3-E1cells. (B) KEGG pathway analysis of downregulated genes in LV-Kaiso cells vs. LV-ctr cells. (C) GO analysis of BP in upregulated genes in Sh-Kaiso MC3T3-E1cells vs. Sh-ctr MC3T3-E1cells. (D) KEGG pathway analysis of upregulated genes in Sh-Kaiso cells vs. Sh-ctr cells. (E) Protein-protein interaction network analysis of the differentially expressed genes enriched in the PI3K/AKT pathway. Size is proportional to the degree of connectivity. BP, biological process; DifGene, differentially expressed gene; BP, biological processes; GO, Gene Ontology; LV, lentivirus; shRNA, short hairpin RNA; Ctr, control; KEGG, Kyoto Encyclopedia of Genes and Genomes.

Itag 10 could partially restore the suppressed Itga10 promoter activity (relative luciferase activity, $0.39 \pm 0.04$ in Itga10-WT vs. $0.50 \pm 0.04$ in Itga10-MUT) (Fig. 4D and E).
To examine the direct role of Itga10 in osteogenesis of MC3T3-Elcells, two shRNAs were used to knock down Itga10 (Sh-Itga10-1 andSh-Itga10-2). RT-qPCR and western blot 

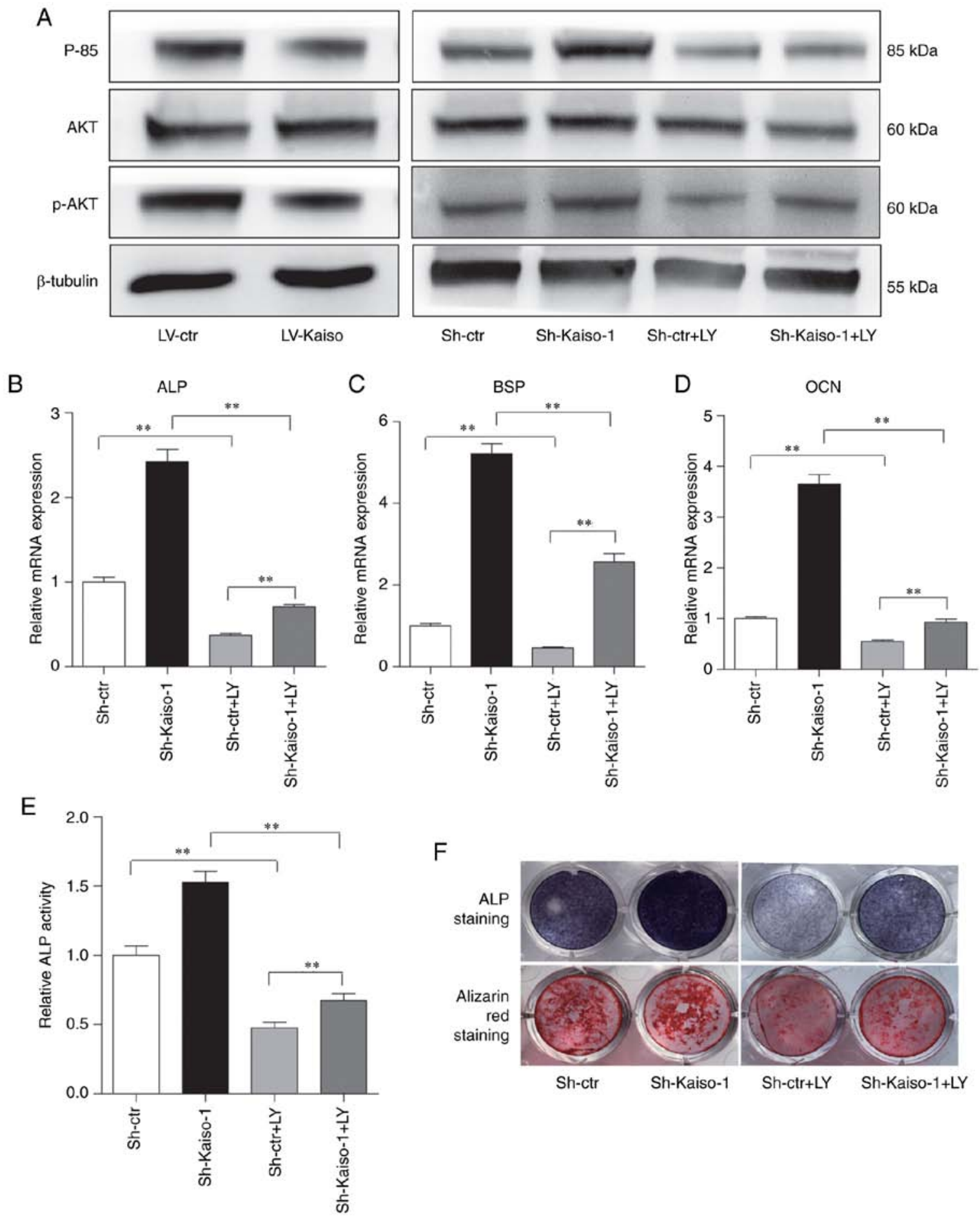

Figure 3. Kaiso regulates osteoblast differentiation by modulating the PI3K/AKT pathway in MC3T3-E1 cells. (A) Western blot analysis was used to detect the protein expression levels of p85, AKT and p-AKT in MC3T3-E1 cells with Kaiso overexpression or Kaiso knockdown. Sh-Kaiso-1 and Sh-ctrl cells were treated with LY, and the expression levels of p85, AKT and p-AKT were examined via western blot analysis. RT-qPCR analysis of (B) ALP, (C) BSP and (D) OCN expression levels in Sh-ctr and Sh-Kaiso transfected MC3T3-E1 cells after 7 days of culture with osteogenic medium in the presence of LY. (E) ALP activity was measured on day 7. (F) ALP staining was performed on day 7, and Alizarin Red S staining was performed on day 21. Data are presented as the mean $\pm \mathrm{SD}(\mathrm{n}=3)$. ${ }^{* *} \mathrm{P}<0.01$. LY, LY294002; RT-qPCR, reverse transcription-quantitative PCR; ALP, alkaline phosphatase; OCN, osteocalcin; BSP, bone sialoprotein; LV, lentivirus; shRNA, short hairpin RNA; Ctr, control; p-, phosphorylated.

analyses identified that both shRNAs led to efficient knockdown of Itga10 in MC3T3-E1 cells (Fig. S4A and B). The results demonstrated that knocking down Itga10 decreased the protein expression levels of p85 and p-AKT (Fig. 4F), as well as the mRNA expression levels of ALP, OCN and BSP (Fig. 4G). Furthermore, the stable Sh-Itga10-1 and Sh-Itga10-2 cell lines had suppressed osteoblast differentiation, as demonstrated by declined ALP activity, ALP staining and mineralized bone nodule formation (Fig. 4H and I).

To examine whether Itga10 serves as a downstream target of Kaiso, cells were transfected with lentivirus expressing Itga 10. The efficiency of lentivirus expressing Itga10 in MC3T3-E1 

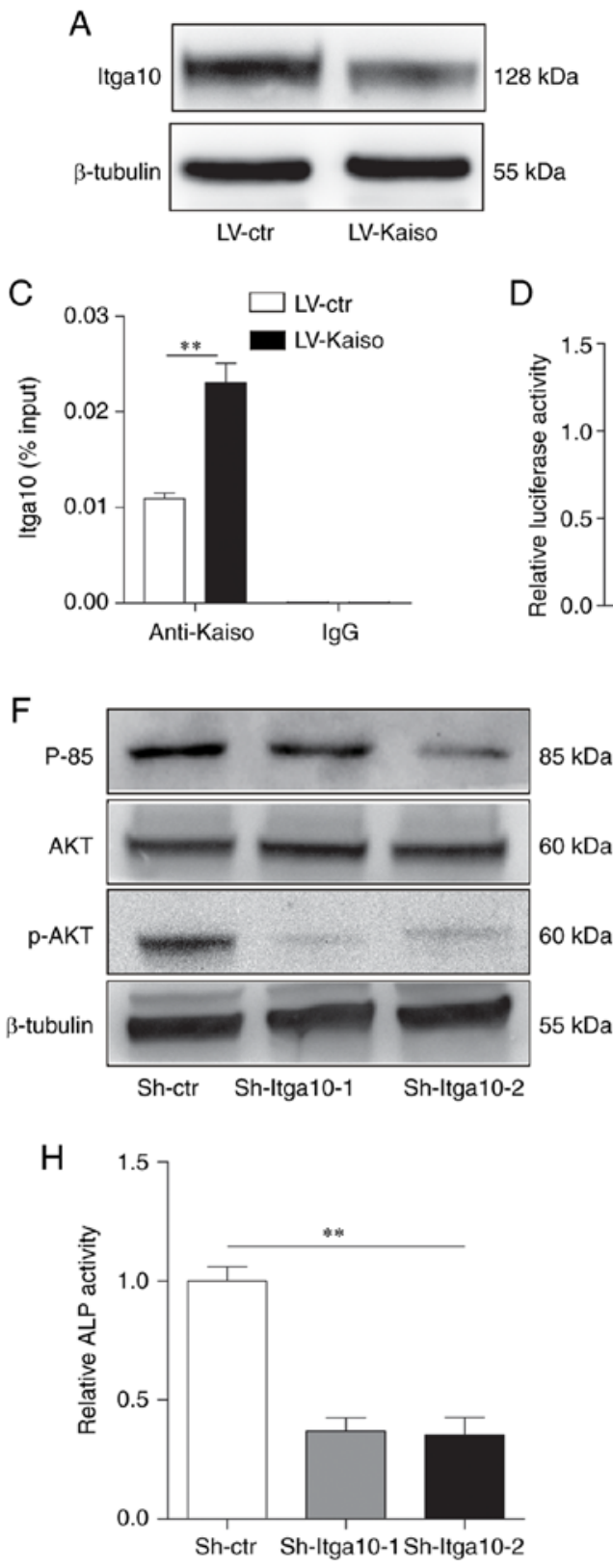
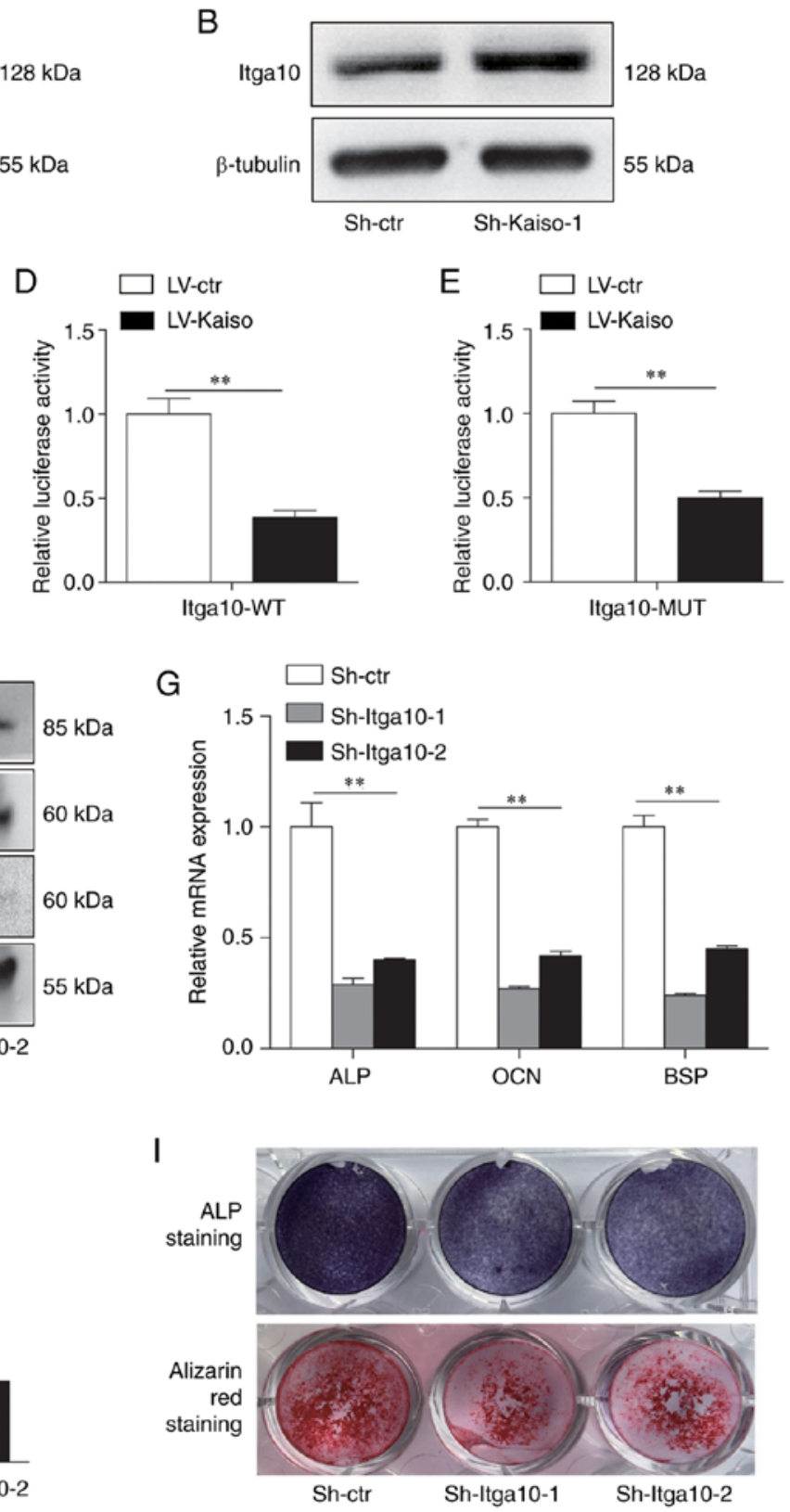

Figure 4. Kaiso regulates the PI3K/AKT pathway via Itga10. Western blot analysis of Itga10 expression in (A) Kaiso-overexpressing or (B) Kaiso-knockdown MC3T3-E1 cells. (C) Chromatin immunoprecipitation analysis of the binding of Kaiso to the promoter of Itga10 in LV-ctr and LV-Kaiso MC3T3-E1 cells. Input DNA and DNA immunoprecipitated with IgG were included as positive and negative controls, respectively. The results are expressed as percentages of the input level. (D) WT and (E) Kaiso binding site-MUT Itga10 promoter-luciferase reporter vectors were co-transfected with Kaiso-overexpression vectors or control vector in MC3T3-E1 cells. Luciferase activity was normalized to Renilla luciferase activity and presented as the fold change to LV-ctr. (F) Expression levels of p85, AKT and p-AKT were determined via western blot analysis in Sh-Itga10-1, Sh-Itga10-2 and Sh-ctr transfectedMC3T3-E1 cells. (G) RT-qPCR analysis of ALP, OCN and BSP expression levels in Sh-ctr and Sh-Kaiso transfected MC3T3-E1 cells after 7 days culture with osteogenic medium. (H) ALP activity was measured on day 7. (I) ALP staining was performed on day 7, and Alizarin Red S staining was performed on day 21. All quantitative data are presented as the mean $\pm \mathrm{SD}(\mathrm{n}=3)$. ${ }^{* *} \mathrm{P}<0.01$. WT, wild-type; MUT, mutant; LV, lentivirus; shRNA, short hairpin RNA; Ctr, control; p-, phosphorylated; ALP, alkaline phosphatase; OCN, osteocalcin; BSP, bone sialoprotein; Itga10, integrin subunit $\alpha 10$; RT-qPCR, reverse transcription-quantitative PCR.

cells was confirmed via western blot analyses and qPCR (Fig. S4C and D). LV-Kaiso cells were infected with a lentivirus expressing Itga10 to produce a stable overexpressing cell line (LV-Kaiso+Itga10). The results demonstrated that overexpression of Itga10 could significantly alleviate the osteoblast inhibitory effects observed in LV-Kaiso-stably transfected cells (LV-ctr vs. LV-Kaiso+Itga10; LV-Kaisovs. LV-Kaiso+Itga10) (Fig. S5). Taken together, these results suggested that Itga10 serves an important role in the Kaiso-mediated regulation of the PI3K/AKT pathway in MC3T3-E1 cells.
Osteogenesis assay in vivo. To investigate the osteogenic potential of LV-Kaiso, Sh-Kaiso and Sh-Itga10 MC3T3-E1 cells in vivo, $\beta$-TCP scaffolds loaded with cells were implanted into the intramuscular pocket of nude mice. Experimental mice were sacrificed using $\mathrm{CO}_{2}$ at the end of the study. After 8 weeks of recovery, newly formed bones were analyzed via $\mu \mathrm{CT}$ and $\mathrm{H} \& \mathrm{E}$ staining. $\mu \mathrm{CT}$ images demonstrated more ectopic bone formation in the Sh-Kaiso group compared with that in the Sh-ctr group by comparison of BMD generated by skyscan 1176. The Kaiso overexpression and Itga10 
A

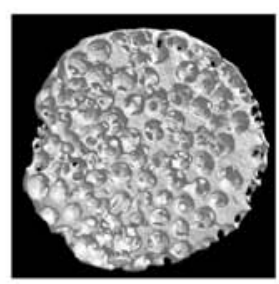

Vehicle

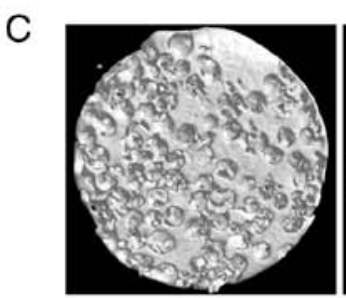

Sh-ctr

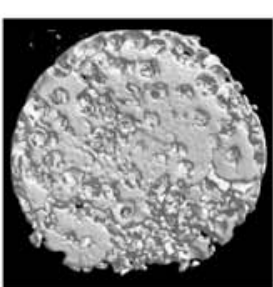

Sh-Kaiso-1
B

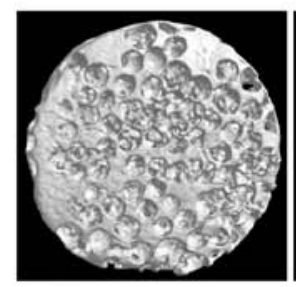

LV-ctr

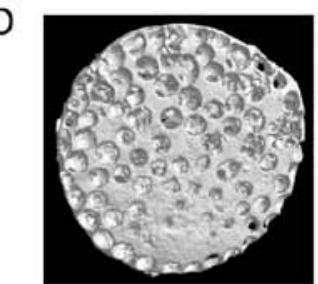

Sh-ctr

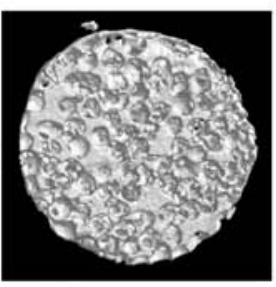

LV-Kaiso

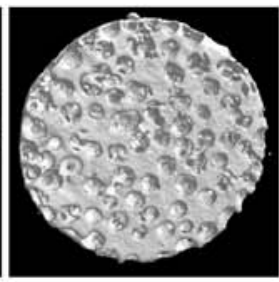

Sh-Itga 10-1

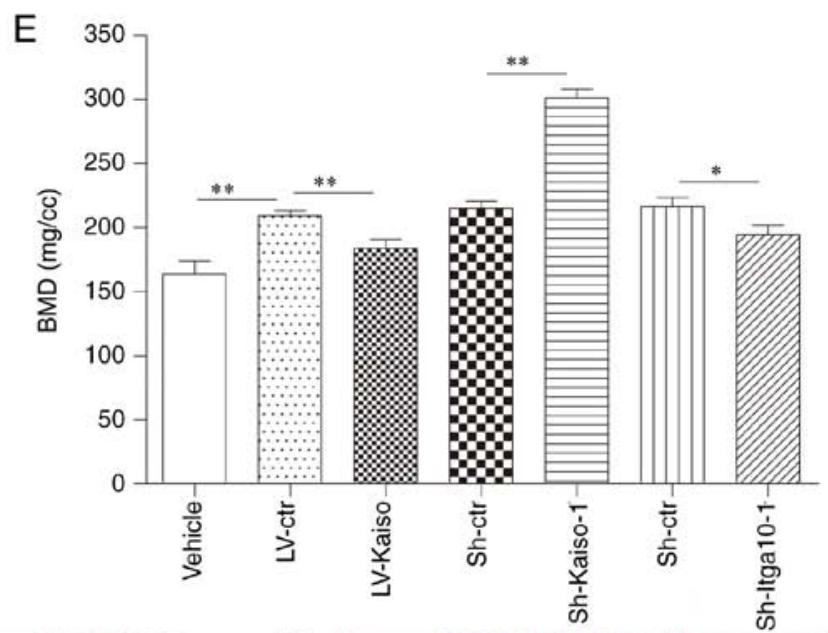

$\mathrm{F}$

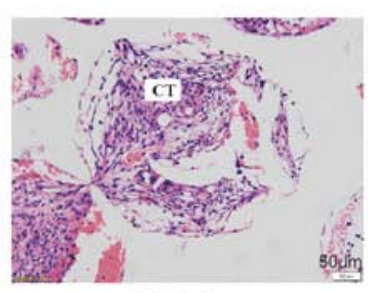

Vehicle

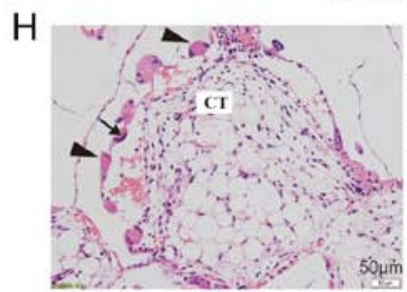

Sh-ctr

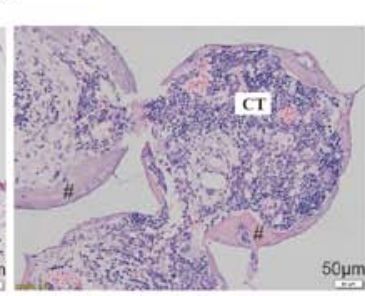

Sh-Kaiso-1

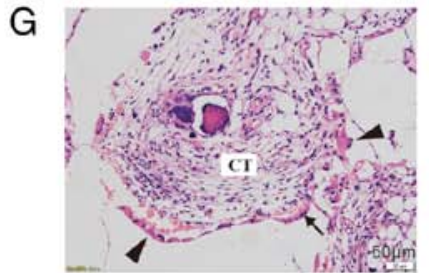

LV-ctr

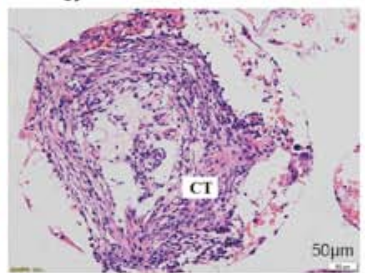

LV-Kaiso

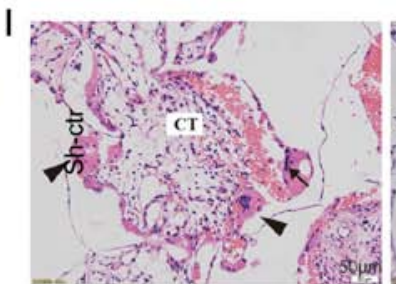

Sh-ctr

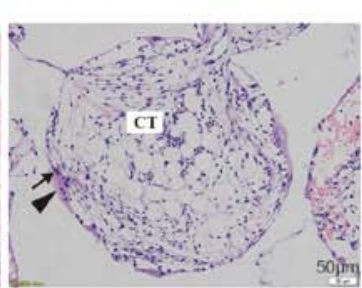

Sh-Itga10-1

Figure 5. In vivo osteogenesis of LV-Kaiso, Sh-Kaiso and Sh-Itga10 infected MC3T3-E1 cells. Representative $\mu$ CT images (magnification, $\mathrm{x} 1$ ) from each group: (A) Vehicle, (B) LV-ctr and LV-Kaiso, (C) Sh-ctr and Sh-Kaiso-1, (D) Sh-ctr and Sh-Itga10-1. (E) BMD of the implants was measured based on $\mu$ CT images. Representative hematoxylin and eosin staining images from each group: (F) Vehicle, (G) LV-ctr and LV-Kaiso, (H) Sh-ctr and Sh-Kaiso-1, (I) Sh-ctr and Sh-Itga10-1. Scale bar, $50 \mu \mathrm{m}$. Thin arrows indicate osteoblast; arrowheads indicate newly formed osteoid; \# indicates newly formed mature bone. Data are presented as the mean $\pm \mathrm{SD}(\mathrm{n}=3)$. ${ }^{*} \mathrm{P}<0.05$ and ${ }^{* *} \mathrm{P}<0.01$. BMD, Bone mineral density; CT, connective tissue; Itga10, integrin subunit $\alpha 10$; LV, lentivirus; shRNA, short hairpin RNA; Ctr, control.

knockdown groups had decreased regenerated bone volume compared with that in the control group (Fig. 5A-D). These results were confirmed via measurement of BMD (Fig. 5E). The results of H\&E staining provided further evidence for the differences in bone formation in the $\beta$-TCP scaffolds from different groups (Fig. 5F-I). In the H\&E staining, the lightly dyed continuous structure marked by ' $\#$ ' in the figure represents newly formed mature bone; the small reddish tissue (indicated by arrowheads) is newly formed osteoid; and the deeply stained nuclei (indicated by arrows), which 
can be seen in the osteoid, are osteoblasts. The Sh-Kaiso group had more newly formed mature bone compared with the other groups.

\section{Discussion}

The present study identified the essential role of Kaiso during osteoblast differentiation in MC3T3-E1 cells, and it was identified that Kaiso regulated this process via the PI3K/AKT pathway by binding to the promoter of Itga10. To the best of our knowledge, this was the first study to demonstrate the potential involvement of Kaiso in osteoblast differentiation and bone formation.

Previous studies have reported that the transcription factor Kaiso functions in various biological processes. For instance, Kaiso regulates the cell cycle viacyclins D1 and E1 (12). Moreover, Kaiso acts as an essential transcriptional factor in the proliferation and migration of cancer cells $(13,14)$, as well as potentiates intestinal tumorigenesis in the murine intestine $(11,15)$. Kaiso also serves a role in vascularization, which is critical in bone formation (23). The zinc finger and BTB domain containing 16 gene, which is also a member of the POZ-zinc finger family, has been reported to regulate osteoblast differentiation $(24,25)$. Furthermore, Kaiso is upregulated in subchondral bone of an early experimental osteoarthritis model based on transcription factors analysis (26). These findings indicate that Kaiso may serve important roles in the pathogenesis, including cartilage destruction or osteophyte formation, of early experimental osteoarthritis. The present study identified the role of Kaiso in osteoblast differentiation. The expression of Kaiso was found to be downregulated during osteoblast differentiation in MC3T3-E1 cells. Gain- and loss-of-function in vitro studies demonstrated that overexpression of Kaiso was sufficient to suppress osteoblast differentiation, whereas gene-specific silencing promoted this process. In accordance with these findings, the present results indicated that Kaiso overexpression decreased bone formation, while Kaiso knockdown increased bone formation in vivo. These findings suggested that Kaiso negatively regulated osteoblast differentiation.

Next, the current study investigated the mechanisms via which Kaiso regulated osteoblast differentiation. The effect of Kaiso on Wnt has been widely studied; however, the present results indicated that Kaiso regulated osteoblast differentiation in a Wnt pathway-independent manner. Previous studies have revealed that the PI3K/AKT pathway is critical during osteoblast differentiation $(27,28)$. In the present study, the enhanced osteoblast differentiation observed in Kaiso-knockdown cells was accompanied by increased p85 and p-AKT expression levels, and these effects were almost completely blocked by the PI3K/AKT pathway inhibitor LY294002. Thus, these findings suggested that the PI3K/AKT pathway may be the target of Kaiso during osteoblast differentiation.

Kaiso is a zinc finger transcriptional factor that contains a BTB/POZ domain for PPI at the N-terminus (12). Previous studies have reported that Kaiso regulates pathological processes by directly binding to the promoters of target genes via the KBS or methylated CpG dinucleotides $(12,29,30)$. To evaluate the potential target via which Kaiso regulated the
PI3K/AKT pathway, the present study focused on Itga10. Integrins are cell surface receptors that interact with several signaling molecules and activate intracellular signaling pathways, such as the PI3K/AKT pathway (31). The roles of integrins in osteoblast differentiation, as well as their influence on the physiology and pathophysiology of cartilage and bone have been widely studied (32-35). Itga10 has been reported to modulate chondrocyte differentiation, and can regulate AKT activity (36-38). The present results suggested that Kaiso binds to the KBS region of the Itga10 promoter and inhibits the expression of Itga10. Moreover, mutation of the KBS in the promoter of the Itga10 gene partially alleviated the inhibition of luciferase reporter activity observed in Kaiso-overexpressing MC3T3-E1 cells. This may be attributed to the existence of other methylated $\mathrm{CpG}$ dinucleotide binding sites. Knocking down Itga10 decreased the activation of the PI3K/AKT pathway and suppressed osteoblast differentiation, while overexpression of Itga10 could significantly alleviate the osteoblast inhibitory effects observed in Kaiso-stably transfected cells. These findings indicated that Kaiso regulated the PI3K/AKT pathway by binding to the Itga10 promoter.

In conclusion, the present study demonstrated that Kaiso regulated osteoblast differentiation in MC3T3-E1 cells via the Itga10/PI3K/AKT signaling pathway. Kaiso and the Itga10/PI3K/AKT signaling pathway may serve an essential role in bone destruction and bone formation, thus representing a novel therapeutic target for related diseases.

\section{Acknowledgements}

Not applicable.

\section{Funding}

This research was supported by the National Nature Science Foundation of China (grant nos. 81601412, 81901654 and 81672126) and the Youth Startup Foundation of Changhai Hospital (grant no. 2018QNA017).

\section{Availability of data and materials}

The analyzed data sets generated during the study are available from the corresponding author on reasonable request.

\section{Authors' contributions}

Study design: WX and CH. Acquisition of data: WT, XF and JL. Analysis and interpretation of data: JL, CW and YX. Drafting the article or revising: WT, JL and XF. All authors read and approved the final manuscript.

\section{Ethics approval and consent to participate}

All animal studies were approved by the Animal Ethics Committee of Changhai Hospital.

\section{Patient consent for publication}

Not applicable. 


\section{Competing interests}

The authors declare that they have no competing interests.

\section{References}

1. Harada S and Rodan GA: Control of osteoblast function and regulation of bone mass. Nature 423: 349-355, 2003.

2. Teitelbaum SL and Ross FP: Genetic regulation of osteoclast development and function. Nat Rev Genet 4: 638-649, 2003.

3. Loi F, Córdova LA, Pajarinen J, Lin TH, Yao Z and Goodman SB Inflammation, fracture and bone repair. Bone 86: 119-30, 2016.

4. Sieper J and Poddubnyy D: Axial spondyloarthritis. Lancet 390 73-84, 2017.

5. Glyn-Jones S, Palmer AJ, Agricola R, Price AJ, Vincent TL, Weinans H and Carr AJ: Osteoarthritis. Lancet 386: 376-387, 2015.

6. Yang TL, Shen H, Liu A, Dong SS, Zhang L, Deng FY, Zhao Q and Deng HW: A road map for understanding molecular and genetic determinants of osteoporosis. Nat Rev Endocrinol 16 : 91-103, 2020

7. Day TF, Guo X, Garrett-Beal L and Yang Y: Wnt/beta-catenin signaling in mesenchymal progenitors controls osteoblast and chondrocyte differentiation during vertebrate skeletogenesis. Dev Cell 8: 739-750, 2005.

8. Franceschi RT, Ge C, Xiao G, Roca H and Jiang D: Transcriptional regulation of osteoblasts. Ann NY Acad Sci 1116: 196-207, 2007.

9. Daniel JM, Spring CM, Crawford HB, Reynolds AB and Baig A: The p120(ctn)-binding partner Kaiso is a bi-modal DNA-binding protein that recognizes both a sequence-specific consensus and methylated CpG dinucleotides. Nucleic Acids Res 30: 2911-2919, 2002 .

10. Buck-Koehntop BA, Stanfield RL, Ekiert DC, MartinezYamout MA, Dyson HJ, Wilson IA and Wright PE: Molecular basis for recognition of methylated and specific DNA sequences by the zinc finger protein Kaiso. Proc Natl Acad Sci USA 109: 15229-15234, 2012.

11. Choi SH, Koh DI, Cho SY, Kim MK, Kim KS and Hur MW: Temporal and differential regulation of KAISO-controlled transcription by phosphorylated and acetylated p53 highlights a crucial regulatory role of apoptosis. J Biol Chem 294 12957-12974, 2019.

12. Pozner A, Terooatea T and Buck-Koehntop B: Cell-specific Kaiso (ZBTB33) regulation of cell cycle through cyclin D1 and cyclin E1. J Biol Chem 291: 24538-24550, 2016.

13. Feng J: Upregulation of MicroRNA-4262 targets Kaiso (ZBTB33) to inhibit the proliferation and EMT of cervical cancer cells Oncol Res 26: 1215-1225, 2018.

14. Pierre CC, Hercules SM, Yates C and Daniel JM: Dancing from bottoms up-roles of the POZ-ZF transcription factor Kaiso in cancer. Biochim Biophys Acta Rev Cancer 1871: 64-74, 2019.

15. Short SP, Barrett CW, Stengel KR, Revetta FL, Choksi YA, Coburn LA, Lintel MK, McDonough EM, Washington MK, Wilson KT, et al: Kaiso is required for MTG16-dependent effects on colitis-associated carcinoma. Oncogene 38: 5091-5106, 2019.

16. Kim SW, Park JI, Spring CM, Sater AK, Ji H, Otchere AA, Daniel JM and McCrea PD: Non-canonical Wnt signals are modulated by the Kaiso transcriptional repressor and p120-catenin Nat Cell Biol 6: 1212-1220, 2004.

17. Liu Y, Dong QZ, Wang S, Xu HT, Miao Y, Wang L and Wang EH: Kaiso interacts with p120-catenin to regulate $\beta$-catenin expression at the transcriptional level. PLoS One 9: e87537, 2014.

18. Baron R and Kneissel M: WNT signaling in bone homeostasis and disease: From human mutations to treatments. Nat Med 19: 179-192, 2013

19. Livak KJ and Schmittgen TD: Analysis of relative gene expression data using real-time quantitative PCR and the 2(-Delta Delta C(T)) method. Methods 25: 402-408, 2001

20. Metwally M, Bayoumi A, Romero-Gomez M, Thabet K, John M, Adams LA, Huo X, Aller R, García-Monzón C, Teresa Arias-Loste M, et al: A polymorphism in the Irisin-encoding gene (FNDC5) associates with hepatic steatosis by differential miRNA binding to the 3'UTR. J Hepatol 70: 494-500, 2019.

21. Guan J, Zhang J, Zhu Z, Niu X, Guo S, Wang Y and Zhang C: Bone morphogenetic protein 2 gene transduction enhances the osteogenic potential of human urine-derived stem cells. Stem Cell Res Ther 6: 5, 2015.
22. Collings AJ and Richards CT: Digital dissection of the pelvis and hindlimb of the red-legged running frog, phlyctimantis maculatus, using diffusible iodine contrast enhanced computed microtomography (DICE $\mu$ CT). PeerJ 7: e7003, 2019

23. Delgado-Bellido D, Fernández-Cortés M, Rodríguez MI, Serrano-Sáenz S, Carracedo A, Garcia-Diaz A and Oliver FJ: VE-cadherin promotes vasculogenic mimicry by modulating kaiso-dependent gene expression. Cell Death Differ 26: 348-361, 2019.

24. Marofi F, Vahedi G, Solali S, Alivand M, Salarinasab S, Zadi Heydarabad M and Farshdousti Hagh M: Gene expression of TWIST1 and ZBTB16 is regulated by methylation modifications during the osteoblastic differentiation of mesenchymal stem cells. J Cell Physiol 234: 6230-6243, 2019.

25. Onizuka S, Iwata T, Park SJ, Nakai K, Yamato M, Okano T and Izumi Y: ZBTB16 as a downstream target gene of osterix regulates osteoblastogenesis of human multipotent mesenchymal stromal cells. J Cell Biochem 117: 2423-2434, 2016.

26. Zhang RK, Li GW, Jiang D, Zhang DW, Yu B and Yang LK: Transcription factors analysis of subchondral bone in early experimental osteoarthritis based on gene expression profiles. Zhongguo Gu Shang 31: 165-169, 2018 (In Chinese).

27. Shen WC, Lai YC, Li LH, Liao K, Lai HC, Kao SY, Wang J, Chuong CM and Hung SC: Methylation and PTEN activation in dental pulp mesenchymal stem cells promotes osteogenesis and reduces oncogenesis. Nat Commun 10: 2226, 2019.

28. Ye C, Zhang W, Hang K, Chen M, Hou W, Chen J, Chen X, Chen E, Tang L, Lu J, et al: Extracellular IL-37 promotes osteogenic differentiation of human bone marrow mesenchymal stem cells via activation of the PI3K/AKT signaling pathway. Cell Death Dis 10: 753, 2019

29. Robinson SC, Klobucar K, Pierre CC, Ansari A, Zhenilo S, Prokhortchouk E and Daniel JM: Kaiso differentially regulates components of the Notch signaling pathway in intestinal cells. Cell Commun Signal 15: 24, 2017.

30. Bassey-Archibong BI, Kwiecien JM, Milosavljevic SB, Hallett RM, Rayner LG, Erb MJ, Crawford-Brown CJ, Stephenson KB, Bédard PA, Hassell JA and Daniel JM: Kaiso depletion attenuates transforming growth factor- $\beta$ signaling and metastatic activity of triple-negative breast cancer cells. Oncogenesis 5: e208, 2016.

31. Hynes RO: Integrins: Bidirectional, allosteric signaling machines. Cell 110: 673-687, 2002.

32. Docheva D, Popov C, Alberton P and Aszodi A: Integrin signaling in skeletal development and function. Birth Defects Res C Embryo Today 102: 13-36, 2014.

33. Hamidouche Z, Fromigué O, Ringe J, Häupl T, Vaudin $P$, Pagès JC, Srouji S, Livne E and Marie PJ: Priming integrin alpha5 promotes human mesenchymal stromal cell osteoblast differentiation and osteogenesis. Proc Natl Acad Sci USA 106 18587-18591, 2009

34. Shen B, Vardy K, Hughes P, Tasdogan A, Zhao Z, Yue R, Crane GM and Morrison SJ: Integrin alpha11 is an osteolectin receptor and is required for the maintenance of adult skeletal bone mass. Elife 8: e42274, 2019.

35. Raines AL, Berger MB, Schwartz Z and Boyan BD: Osteoblasts grown on microroughened titanium surfaces regulate angiogenic growth factor production through specific integrin receptors. Acta Biomater 97: 578-586, 2019.

36. Varas L, Ohlsson LB, Honeth G, Olsson A, Bengtsson T, Wiberg C, Bockermann R, Järnum S, Richter J, Pennington D, et al: Alpha10 integrin expression is up-regulated on fibroblast growth factor-2-treated mesenchymal stem cells with improved chondrogenic differentiation potential. Stem Cells Dev 16: 965-978, 2007.

37. Bengtsson T, Aszodi A, Nicolae C, Hunziker EB, LundgrenAkerlund $\mathrm{E}$ and Fässler R: Loss of alpha10betal integrin expression leads to moderate dysfunction of growth plate chondrocytes. J Cell Sci 118: 929-936, 2005.

38. Okada T, Lee AY, Qin LX, Agaram N, Mimae T, Shen Y, O'Connor R, López-Lago MA, Craig A, Miller ML, et al: Integrin- $\alpha 10$ dependency identifies RAC and RICTOR as therapeutic targets in high-grade myxofibrosarcoma. Cancer Discov 6 : $1148-1165,2016$

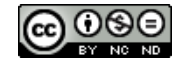

This work is licensed under a Creative Commons Attribution-NonCommercial-NoDerivatives 4.0 International (CC BY-NC-ND 4.0) License. 